\title{
Pain characteristics and countermeasures to improve intraoperative pain for patients with temporomandibular joint disorder during treatment of orthodontics
}

\author{
Junsheng $\mathrm{LIN}^{1}$, Wei ZHANG² , Renfa LAI ${ }^{1 *}$ (D)
}

\begin{abstract}
To explore and analyze the pain characteristics and countermeasures to improve intraoperative pain for patients with temporomandibular joint disorder during treatment of orthodontics. The patients were randomly divided into control group and the observation group. The control group received routine nursing. It is found in the study that the occurrence of pain in orthodontic treatment is accompanied by certain regular changes, and there is little difference between the proportion of masticatory pain and occlusal pain. In the observation group, the total clinical effective rate increased after nursing $(\mathrm{P}<0.05$. The VAS pain score of the observation group was significantly lower than that of the control group $4 \mathrm{~h}$ and $8 \mathrm{~h}$ after nursing intervention was implemented, and the duration of pain symptoms was significantly shorter than that of the control group, with significant statistical difference between the two groups $(\mathrm{P}<0.05)$. The targeted nursing intervention for patients with temporomandibular joint disorder undergoing orthodontic treatment based on their characteristics will shorten the pain time of patients effectively and improve the treatment effect, maintaining significant application value.
\end{abstract}

Keywords: temporomandibular joint disorder; orthodontic treatment; pain characteristics; pain nursing.

Practical Application: The targeted nursing intervention based the characteristics of patients with temporomandibular joint disorder undergoing orthodontic treatment.

\section{Introduction}

Temporomandibular joint disorder (TMD) is the most common disease of oral and maxillofacial region, which is mainly manifested in clinical symptoms such as joint pain, mandibular movement disorder and joint popping during exercise. It occurs frequently to young and middle-aged people and has a serious impact (Goldstein et al., 2019) on the daily life of patients. Currently, this disease is mainly treated by drugs, surgical operation, nerve stimulation and other methods, and orthodontic treatment has positive significance (Yokoyama et al., 2018) in helping patients regain effective oral and maxillary system functions and oral tissue health. However, as a common complication in orthodontic treatment, pain affects directly the patients' acceptance and coordination of the treatment plan to a certain extent, and even causes fear, thus prolonging the return time of treatment, and thereby affecting the surgical effect. Therefore, some effective nursing methods should be adopted to alleviate pain symptoms and ensure the smooth treatment (Zhang et al., 2017) in orthodontic treatment. This study is to explore and analyze the pain characteristics and countermeasures to improve intraoperative pain for patients with TMD during treatment of orthodontics.

\section{Materials and methods}

\subsection{General data}

The 120 patients with temporomandibular joint disorders who suffered from pain during orthodontic treatment in our hospital since March 2018 till March 2019 were selected as the study objects and divided into two groups randomly; In the control group, there were 25 men and 35 women, aged from 18 to 35 years, with an average age of $(27.5 \pm 7.5)$ years. The disease course was 1 to 4 months, with an average course of (3.0 \pm 1.0$)$ months, unilateral: 41 cases, bilateral: 19 cases, under routine nursing. In the observation group, there were 24 males and 36 females, with an average age of $(28.0 \pm 7.0)$ years from 17 to 35 years, a course of disease of 1 to 3 months, an average course of disease of $(2.5 \pm 0.5)$ months, unilateral: 41 cases, bilateral: 18 cases, and targeted nursing was given according to their pain characteristics. All families gave informed consent on the two groups of objects to be studied, approved by the Ethics Committee of our institute. There was no significant difference between the groups in terms of gender, age, course of disease, location of onset and etc. general clinical data $(\mathrm{P}>0.05)$, indicating comparability. The study was approved by our hospital. Informed consent was obtained.

\subsection{Inclusion and exclusion criteria}

Inclusion Criteria: (1) All the selected patients were consistent with the diagnostic criteria stated in Diagnosis of Oral and Maxillofacial Medical Imaging (Ma, 2012); (2) Accompanied with joint popping, joint or periarticular muscle group pain, abnormal mandibular movement; (3) Informed consent signed by patients or family members. 
Exclusion Criteria: (1) Organic changes observed through temporomandibular arthrography or CT; (2) Inconsistent with indications of orthodontic surgery; (3) Accompanied by neurological disease.

\subsection{Methods}

Patients in the control group received routine nursing. During the operation, patients would be observed on the changes in facial color and facial expression, asked whether there was pain or discomfort, comforted and encouraged by words, and explained to them that the duration of pain symptoms was relatively short, so as to improve the pain tolerance for patients.

The observation group was given targeted nursing based on their pain characteristics, and paid close attention to the postoperative pain generation and changes of patients through inquiry, questionnaire, clinical observation, pain evaluation and other methods; The general data of patients were collected, including gender, age, previous medical history, treatment time, type of orthotics used, anxiety status and severity of the disease and etc., so as to understand the patients' tolerance to pain, current mental states and emotional changes, and let the patient describe the pain experience. Then, Eysenck Personality Questionnaire (EPQ) was used to assess the personality characteristics and emotional types of patients, and then pain characteristics were further analyzed, and specific intervention was given according to different pain nursing methods. (1) Mental Nursing: before orthodontic treatment, strengthened health education on patients, intraoperative considerations and pain in orthodontic treatment characteristics, performance and told the safety and necessity of orthodontic surgery, illustrated successful cases of patients, communicated with each other, put down the burden in the heart, let patients eliminate inner feelings, calm, peaceful state of mind. Give full understanding to intraoperative patients and build a good doctor-patient relationship, pay attention to see their eyes, face changes, even asking whether patients with pain symptoms, and give more encouragement and comfort, cheer for patients to look in the eyes and gestures, or physical contact way, such as a handshake to make patients feel at ease, and through the chat transfer patient attention, increase the pain threshold; (2) Nursing with Music: the head nurse would organize nurses to compile a "music library", download light music, pop music, classical music, piano music, country music, western slow music and etc., playing music on the basis of patient preferences in the book, recommend patients to choose slow and soft music, such as The Sea, Song Tao Sound, Raindrop, Sea Wave and etc. natural light music; At the same time, pay attention to the quiet surrounding environment when playing music for the patient during the operation, avoid the interference of telephone or visitors, and play the volume to the patient's appropriate, to ensure the patient being fully engaged in the music melody; (3) Cognition Relaxation Nursing: choose a soothing and gentle song from the music library as the background music, help the patient to take a comfortable posture before surgery, tell them to close their eyes, keep natural breathing, follow the unified audio design for visual imagination, make the head, shoulder and neck, arms, body, legs and feet relax in turn; Each cycle lasted for 20 min until the operation was completed; (4) Control of Proper
Orthodontics Force: in the treatment, doctors should pay more attention to the control of orthodontic force. If orthodontic force is too large, it may lead to periodontal damage and increase the pain response. Therefore, doctors should pay attention to the emotional changes of patients, adjust and control the surgical force.

\subsection{Evaluation criteria}

The pain characteristics of the selected patients in orthodontic treatment were analyzed, including pain types such as masticatory pain and occlusal pain; Grade I was pain free; Grade II was mild pain, which had no effect on the patient's mood; Level III is moderate pain, which has a slight effect on mood; Grade IV pain is severe and unbearable, affecting the patient's mood.

Comparing the treatment effect of the two groups of patients after different nursing intervention was implemented, if the patient's mouth was opened normally after nursing, there was no pop, the pain of chewing was eliminated, and there was no tenderness in the joint area, it meant being cured; If the opening degree of patient after nursing was significantly improved, there was slight elasticity, the opening degree was $30 \mathrm{~mm}$ or above, accompanied by mild tenderness in the joint area and chewing pain, it meant being markedly effective; If the patient's opening degree, joint pain and abnormal mouth opening were improved after nursing, and the symptoms of joint popping were alleviated, it meant being effective; If the mouth opening, sound, mouth chewing pain and joint tenderness symptoms of the patient after nursing had no significant changes, then it meant being invalid; The total effective rate $=($ cured + markedly effective + effective $) /$ total cases ${ }^{\star} 100.0 \%$ (Fu \& Ji, 2017).

The pain symptoms improvement effect of patients in two groups was observed before and $2 \mathrm{~h}, 4 \mathrm{~h}, 8 \mathrm{~h}$ after implementation of nursing intervention. The visual analogue scale (VAS) was used to assess the patient's own pain degree, with $0 \sim 10$ points prevailing, where 0 point meant painless, 10 points meant severe pain. The higher score, the more serious (HäggmanHenrikson et al., 2018) the pain in patients.

The duration of pain after nursing intervention was compared between the two groups.

\subsection{Statistical methods}

The data obtained from this study were included in SPSS20.0 software for statistical analysis, $\mathrm{x}^{2}$ test for enumeration data and $t$ for measurement data, and $\mathrm{P}<0.05$ showed statistically significant difference.

\section{Results}

\subsection{Occurrence characteristics of pain in all patients undergoing orthodontic treatment}

It is found in the study that the occurrence of pain in orthodontic treatment is accompanied by certain regular changes, and there is little difference between the proportion of masticatory pain and occlusal pain, while the proportion of grade II pain patients and pain patients with an average occurrence time of $24 \mathrm{~h} \sim 48 \mathrm{~h}$ is higher, and the proportion of pain grade I, III and 
IV patients and pain with an average occurrence time of 4-5 h is lower, as shown in Table 1 and Figure 1.

\subsection{Comparison of treatment effect of the two groups after nursing}

The data of the two groups showed that the operation effect of the observation group was improved, and the pain symptoms were significantly relieved after nursing intervention. The total effective rate of the observation group was $91.7 \%$, significantly higher than that of the control group, $78.3 \%$. The difference between the two groups was statistically significant $(\mathrm{P}<0.05)$, as shown in Table 2 and Figure 2.

\subsection{Improvement of VAS pain symptoms of patients in the two groups before/after nursing}

The analysis on the data between the two groups showed that the two groups had no significant pain symptoms before nursing,

Table 1. Occurrence characteristics of pain in all patients undergoing orthodontic treatment (\%).

\begin{tabular}{lcl}
\hline \multicolumn{2}{l}{ Pain Characteristics } & Cases $(\mathrm{n})$ \\
\hline $\begin{array}{l}\text { Type of } \\
\text { pain }\end{array}$ & Masticatory pain & $64(53.3)$ \\
& Occlusal pain & $56(46.7)$ \\
Grade of & Grade I & $22(18.3)$ \\
pain & Grade II & $50(41.7)$ \\
& Grade III & $38(31.7)$ \\
Mean time & Grade IV & $10(8.3)$ \\
of pain & $4 \sim 5 \mathrm{~h}$ & $41(34.2)$ \\
\hline
\end{tabular}

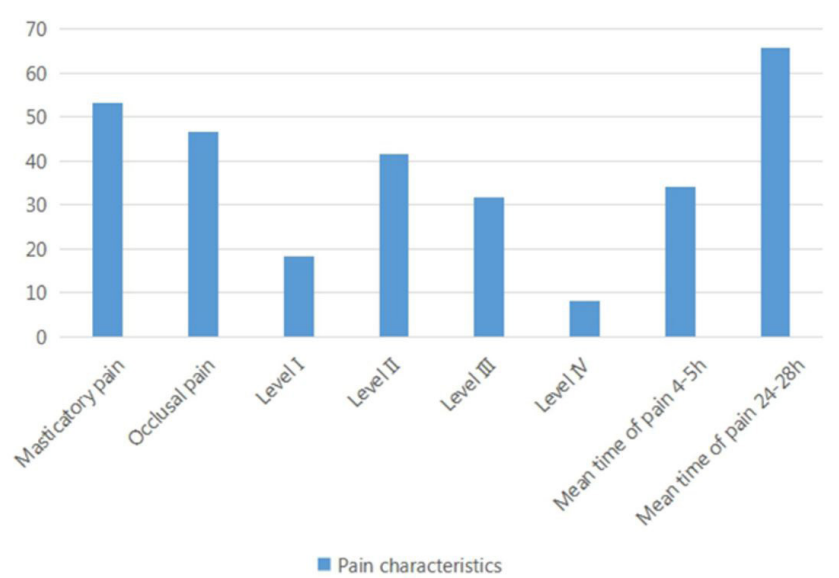

Figure 1. Pain characteristics of patients in orthodontic treatment. and the difference was not statistically significant $(P>0.05)$; After nursing, the patients' pain symptoms were improved, but the difference between the two groups was not statistically significant $2 \mathrm{~h}$ after nursing $(\mathrm{P}>0.05)$, while the VAS pain score of the observation group was significantly lower than that of the control group $4 \mathrm{~h}$ and $8 \mathrm{~h}$ after nursing intervention, and the difference between the two groups was statistically significant $(\mathrm{P}<0.05)$, as shown in Table 3.

\subsection{Duration of pain symptoms in both groups after nursing}

The data compared between the two groups (referred in Table 4) showed that, the duration of pain symptoms in the observation group after being treated with targeted nursing was significantly shorter than that in the control group, and the difference between the two groups was statistically significant $(\mathrm{P}<0.05)$.

\section{Discussion}

Temporomandibular joint, also known as temporomandibular joint, is composed of articular capsule and articular ligament around the articular disc between the condylar process of the mandibular joint and the concave mandibular joint of the temporal bone, while the articular disc is composed of fibrous cartilage. In anatomical morphology, it is one of the most complex joint tissues in human body, and the joint cavity is usually divided into two layers at top and bottom. In front of the joint concave is the joint nodule of the zygomatic process of the temporal bone, which plays an important role (Zegwaard et al., 2017) in preventing excessive sliding dislocation of the condyle when

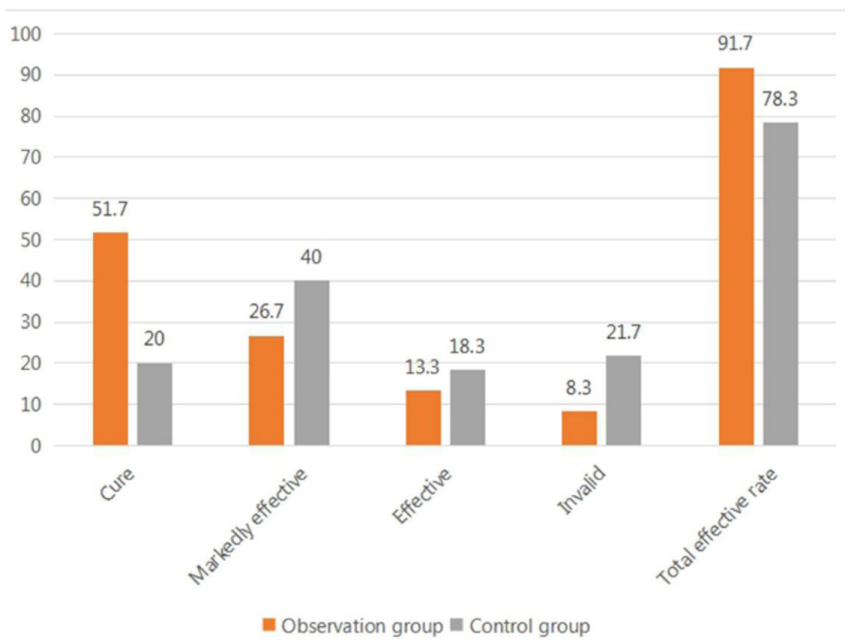

Figure 2. Comparison of treatment effect between the two groups.

Table 2. Comparison of treatment effect of the two groups after nursing (\%).

\begin{tabular}{lcccccc}
\hline Group & Cases $(\mathrm{n})$ & Cured & Markedly Effective & Effective & Invalid & Total Effective Rate \\
\hline Control group & 60 & $12(20.0)$ & $24(40.0)$ & $11(18.3)$ & $13(21.7)$ & $47(78.3)$ \\
Observation group & 60 & $31(51.7)$ & $16(26.7)$ & $8(13.3)$ & $5(8.3)$ & $55(91.7)$ \\
$X^{2}$ & - & 21.848 & 3.979 & 0.940 & 7.042 & 7.042 \\
$p$ & - & 0.000 & 0.046 & 0.332 & 0.008 & 0.008 \\
\hline
\end{tabular}


Table 3. Improvement of VAS pain symptoms of patients in the two groups before/after nursing ( $\pm \mathrm{s})$.

\begin{tabular}{lccccc}
\hline \multicolumn{1}{c}{ Group } & Cases $(\mathrm{n})$ & Before Nursing & 2h after Nursing & 4h after Nursing & 8h after Nursing \\
\hline Control group & 60 & $6.8 \pm 1.4$ & $6.1 \pm 1.2$ & $5.0 \pm 0.9$ & $4.1 \pm 0.7$ \\
Observation group & 60 & $7.0 \pm 1.5$ & $5.8 \pm 1.0$ & $3.8 \pm 0.6$ & $3.0 \pm 0.5$ \\
$t$ & - & 0.755 & 1.488 & 9.295 & 9.905 \\
$p$ & - & 0.452 & 0.140 & 0.000 & 0.000 \\
\hline
\end{tabular}

Table 4. Duration of pain symptoms in both groups after nursing $(\mathrm{x} \pm \mathrm{s})$.

\begin{tabular}{lcc}
\hline Group & Cases $(\mathrm{n})$ & Duration of Pain $(\mathrm{d})$ \\
\hline Control group & 60 & $4.1 \pm 1.0$ \\
Observation group & 60 & $1.8 \pm 0.4$ \\
$t$ & - & 16.542 \\
$p$ & - & 0.000 \\
\hline
\end{tabular}

the mouth is opened. Meanwhile, the temporomandibular joint is the only flexbile joint in the maxillofacial region, which can perform rotational movement and sliding movement, and the left and right joint is coordinated and unified, and participate in chewing, swallowing, speech and other functions. There are many types of temporomandibular joints, such as temporomandibular joint disorder, temporomandibular joint dislocation, temporomandibular joint ankylosis, obstructive apnea syndrome and etc. (Santos et al., 2018). Temporomandibular joint disorder syndrome is a common disease in clinical practice, which is characterized by high incidence, mainly occurring in young and middle-aged women, initially occurring in one side, and gradually affecting both sides as the disease progresses, with a duration of several years or more and strong recurrence (Zegwaard et al., 2017); Although temporomandibular joint disorder syndrome is not easy to cause joint ankylosis, its etiology is complex and its pathological mechanism has not been fully elucidated. It's believed in the mainstream studies that this disease is associated with psychiatric disorders, bilateral temporomandibular joint dysplasia, unilateral chewing, longterm chewing of hard food and other factors (Santos et al., 2018; Dugashvili et al., 2017). At present, it is clinically believed that temporomandibular joint disorder is caused by a combination of multiple factors, including mental factors, jaw biting factors, immune factors, joint overburden, anatomical factors (small condyle, over-active joint prone to dislocation and etc.) (Nkhoma et al., 2018); The clinical stage of this disease mainly includes three parts, that is, functional change stage, structural change stage and joint organic destruction stage. The clinical symptoms are usually abnormal mandibular movement, local pain and local bounce; In addition, there can be spring sound, broken sound and fricative (Hwang and Park, 2018) when it gets abnormal.

At present, the treatment methods for temporomandibular joint disorder are usually selected based on the pathogenesis and development stage, and the common types include conservative treatment, disc repair, disc reduction, implantable substitute therapy and etc. Orthodontic treatment is a new treatment developed in recent years, which is ideal for temporomandibular joint disorder syndrome and maintains the advantages of short pain cycle and low intensity and etc. (Paulino et al., 2018). According to clinical experience, the average occurrence time of periodontal pain is about $4 \sim 5 \mathrm{~h}$ after the patient is equipped with fixed orthodontic device and the first bow wire is placed, and the pain degree will reach the peak within $1 \sim 2 \mathrm{~d}$, which usually needs to be alleviated $72 \mathrm{~h}$ later. It is found in the studies of Bertoli et al. (2018). that the negative emotions such as tension, anxiety and fear can stimulate the secretion of adrenalin by the sympathetic nervous system to a certain extent, thus affecting blood pressure and heart rate in the course of orthodontic treatment. It is proposed in the study of Kim et al. (2018) that psychological intervention measures could effectively alleviate the adverse mood and improve the pain effect for temporomandibular joint disorder syndrome. In addition, it is believed by Banerjee et al. (2018) that the patients' circulatory system could be regulated effectively to promote their heart rate to be normal through parasympathetic excitation and sympathetic suppression. It is believed in the study of Al-Balbeesi et al. (2016) that there would demonstrate certain difference in the clinical manifestations of pain after placing the bow wire in orthodontic treatment for patients with temporomandibular joint disorder syndrome, and appropriate orthodontic force control could improve the pain effect effectively. Besides, Minghui et al. (2017) further proposed that there was no difference in the degree of periodontal pain after placing the first two bow wires, but the duration of pain after placing the second bow wire was shorter, during orthodontic treatment. Meanwhile, it was found by Badrov et al. (2017) that the degree of orthodontic pain in adolescents was significantly higher than that in adults, while the degree of pain in females was lower than that in males, and the duration was relatively longer. It was suggested in the studies of Wang et al. (2017) that the pain manifestations of patients with temporomandibular joint disorder syndrome were mainly of medium and low intensity, and there were certain differences between the acute and chronic phases and between men and women, which could affect the chewing and psychological state of patients. The chronic phase mainly showed dull pain, wood pain and swelling pain. As the mentioned above, pain in patients with temporomandibular joint disorder syndrome during orthodontic treatment should be combined with its pain characteristics and corresponding treatment methods should be selected. In this study, effective psychological intervention was carried out on the observation group of patients, attention was diverted by music nursing, corresponding cognitive relaxation nursing was given, and appropriate orthodontic force control was adopted to improve the pain control effect and improve the negative emotions of patients effectively. 


\section{Conclusion}

In conclusion, the targeted nursing intervention based the characteristics of patients with temporomandibular joint disorder undergoing orthodontic treatment according to the pain symptoms will shorten the pain duration for patients and improve treatment effect, with significant application value.

\section{References}

Al-Balbeesi, H. O., Bin Huraib, S. M., AlNahas, N. W., AlKawari, H. M., Abu-Amara, A. B., Vellappally, S., \& Anil, S. (2016). Pain and distress induced by elastomeric and spring separators in patients undergoing orthodontic treatment. Journal of International Society of Preventive \& Community Dentistry, 6(6), 549-553. http://dx.doi. org/10.4103/2231-0762.195519. PMid:28032047.

Badrov, J., Gašpar, G., Tadin, A., Galić, T., Kalibović Govorko, D., Gavić, L., Badrov, R., \& Galić, I. (2017). Prevalence and characteristics of congenitally missing permanent teeth among orthodontic patients in Southern Croatia. Acta Stomatologica Croatica, 51(4), 290-299. http://dx.doi.org/10.15644/asc51/4/3. PMid:29872234.

Banerjee, S., Banerjee, R., Shenoy, U., Agarkar, S., \& Bhattacharya, S. (2018). Effect of orthodontic pain on quality of life of patients undergoing orthodontic treatment. Indian Journal of Dental Research, 29(1), 4-9. http://dx.doi.org/10.4103/ijdr.IJDR_113_16. PMid:29442079.

Bertoli, F. M. P., Bruzamolin, C. D., Pizzatto, E., Losso, E. M., Brancher, J. A., \& de Souza, J. F. (2018). Prevalence of diagnosed temporomandibular disorders: a cross-sectional study in Brazilian adolescents. PLoS One, 13(2), e0192254. http://dx.doi.org/10.1371/ journal.pone.0192254. PMid:29420573.

Dugashvili, G., Van den Berghe, L., Menabde, G., Janelidze, M., \& Marks, L. (2017). Use of the universal pain assessment tool for evaluating pain associated with TMD in youngsters with an intellectual disability. Medicina Oral, Patologia Oral y Cirugia Bucal, 22(1), e88-e94. PMid:27918746.

Fu, S. J., \& Ji, X. R. (2017). Effect of traditional Chinese medicine-based nursing intervention on gastrointestinal function, psychological mood, and pain in patients after surgery for lumbar disc herniation. World Chinese Journal of Digestology, 25(25), 2296. http://dx.doi. org/10.11569/wcjd.v25.i25.2296.

Goldstein, E., McDonnell, C., Atchley, R., Dorado, K., Bedford, C., Brown, R. L., \& Zgierska, A. E. (2019). The impact of psychological interventions on posttraumatic stress disorder and pain symptoms: a systematic review and meta-analysis. The Clinical Journal of Pain, 35(8), 703-712. http://dx.doi.org/10.1097/AJP.0000000000000730. PMid:31145146.

Häggman-Henrikson, B., Wiesinger, B., \& Wänman, A. (2018). The effect of supervised exercise on localized TMD pain and TMD pain associated with generalized pain. Acta Odontologica Scandinavica, 76(1), 6-12. http://dx.doi.org/10.1080/00016357.2017.1373304. PMid:28870137.
Hwang, S. H., \& Park, S. G. (2018). Experience of orthodontic treatment and symptoms of temporomandibular joint in South Korean Adults. Iranian Journal of Public Health, 47(1), 13-17. PMid:29318112.

Kim, Y. H., Park, Y. G., Han, K. D., Vu, D., Cho, K. H., \& Lee, S. Y. (2018). Prevalence of tinnitus according to temporomandibular joint disorders and dental pain: The Korean National Population-based Study. Journal of Oral Rehabilitation, 45(3), 198-203. http://dx.doi. org/10.1111/joor.12604. PMid:29314140.

Ma XC. (2012). Image diagnosis of oral and maxillofacial medicine. People's Medical Publishing House.

Minghui, P., Jing, K., \& Xiao, D. (2017). Effect of body image in adolescent orthodontic treatment. West China Journal of Stomatology, 35(5), 489-493. PMid:29188643.

Nkhoma, K., Norton, C., Sabin, C., Winston, A., Merlin, J., \& Harding, R. (2018). Self-management interventions for pain and physical symptoms among people living with HIV: a systematic review of the evidence. JAIDS Journal of Acquired Immune Deficiency Syndromes, 79(2), 206-225. http://dx.doi.org/10.1097/QAI.0000000000001785. PMid:30212435.

Paulino, M. R., Moreira, V. G., Lemos, G. A., Silva, P. L. P. D., Bonan, P. R. F., \& Batista, A. U. D. (2018). Prevalence of signs and symptoms of temporomandibular disorders in college preparatory students: associations with emotional factors, parafunctional habits, and impact on quality of life. Ciência \& Saúde Coletiva, 23(1), 173-186. http:// dx.doi.org/10.1590/1413-81232018231.18952015. PMid:29267822.

Santos, T. G. D., Miranda, I. A. S., Nygaard, C. C., Schreiner, L., Castro, R. A., \& Haddad, J. M. (2018). Systematic review of oral therapy for the treatment of symptoms of bladder pain syndrome: the Brazilian guidelines. Revista Brasileira de Ginecologia e Obstetrícia, 40(2), 96-102. http://dx.doi.org/10.1055/s-0037-1609049. PMid:29241263.

Wang, L., Zhou, Q., Tian, H., \& Zhao, J. (2017). Analysis on the laws of acupoint selection and therapeutic operations with acupuncture based on the characteristics of postherpetic neuralgia. Chinese Acupuncture \& Moxibustion, 37(4), 429-431. PMid:29231598.

Yokoyama, Y., Kakudate, N., Sumida, F., Matsumoto, Y., Gordan, V. V., \& Gilbert, G. H. (2018). Dentist's distress in the management of chronic pain control: the example of TMD pain in a dental practicebased research network. Medicine, 97(1), e9553. http://dx.doi. org/10.1097/MD.0000000000009553. PMid:29505535.

Zegwaard, M. I., Aartsen, M. J., Grypdonck, M. H., \& Cuijpers, P. (2017). Trust: An essential condition in the application of a caregiver support intervention in nursing practice. BMC Psychiatry, 17(1), 47. http://dx.doi.org/10.1186/s12888-0171209-2. PMid:28148235.

Zhang, J. X., Wang, J. C., Han, L., Cao, X., \& Shields, L. (2017). Tools to assess risk of bias in systematic reviews of nursing intervention in China: global implications of the findings. Nursing Outlook, 65(4), 380-391. http://dx.doi.org/10.1016/j.outlook.2016.11.004. PMid:28024756. 